\title{
New Acceptance Sampling Plans Based on Percentiles for Type-II Generalized Log Logistic Distribution
}

\author{
G. Srinivasa Rao ${ }^{1, *}$, K. Rosaiah ${ }^{2}$, S. V. S. V. S. V. Prasad ${ }^{3}$ \\ ${ }^{1}$ Department of Statistics, The University of Dodoma, P.O.Box: 259, Tanzania \\ ${ }^{2}$ Department of Statistics, Acharya Nagarjuna University, Guntur - 522 510, India \\ ${ }^{3}$ Reserve Bank of India, Nagpur-440001, India \\ *Corresponding author: gaddesrao@gmail.com
}

Received April 27, 2019; Revised June 05, 2019; Accepted June 21, 2019

\begin{abstract}
This article describes the development of an acceptance sampling plan based on percentiles for Type-II generalized log-logistic distribution (TGLLD) introduced by Rosaiah et. al. [1]. The plan is developed by considering the lifetime percentiles as a variable and the life test will be terminated at a pre-specified time. The objective of the test is to determine the minimum sample size required to achieve a specific lifetime percentile at an acceptable level of consumer and producer risks. Determined the OC values and are presented along with producer risks. The sustainability of the plan is illustrated with real data set.
\end{abstract}

Keywords: Type-II generalized log-logistic distribution, acceptance sampling, truncated life test

Cite This Article: G. Srinivasa Rao, K. Rosaiah, and S. V. S. V. S. V. Prasad, "New Acceptance Sampling Plans Based on Percentiles for Type-II Generalized Log Logistic Distribution." American Journal of Applied Mathematics and Statistics, vol. 7, no. 4 (2019): 131-137. doi: 10.12691/ajams-7-4-2.

\section{Introduction}

In Statistical quality control, acceptance sampling plan has proven its importance and finds its existence in many industry sectors. As we know, while accessing the quality of product no one will prefer to go for a $100 \%$ inspection. In such scenario, acceptance sampling is a key alternative which will helps in decision making whether to accept or reject a group of products or items based on the quality observed in a sample. Acceptance sampling plans got popularized by Dodge and Roming and originally applied by the U.S military to the testing of bullets during World War-II. It is a method of inspecting a sample of products to decide whether the product lot to be accepted or not, based on the results obtained. If the number of failures during the test time does not exceed the acceptance number then the lot is accepted.

In order to have the control over the time and cost, the concept of truncated life tests is originated and it was overcame difficulties in the traditional life testing, wherein one has to wait till either the mean life of products is greater than or less than the specified value so as to make a decision whether the lot to be accepted or rejected. Hence in order to achieve the objective of minimizing the time and cost of the test, one may opt for a truncated life test wherein it can be determined the smallest sample size to ensure the certain mean life of products at a pre-specified test termination time $t_{0}$ and a given acceptance number c. In a usual life testing, the test is terminated at a pre-specified time $t_{0}$ and record the number of failures. It is imperative to specify lower confidence limit of the mean life. Then it is to establish pre-determined mean life at a given probability $\alpha$, which is nothing but an indicator of consumer confidence. The lot may be accepted if the observed number of failures at the end of pre-specified time $t_{0}$ does not exceed a given number c. On other hand, the life test is concluded at the time $(\mathrm{c}+1)^{\text {th }}$ failure is observed or at the pre-specified test termination time $t_{0}$. If we refer the development cycle of acceptance sampling, distinguished authors paid their attention in developing the different methods of testing viz. Epstein [2], Sobel and Tischendrof [3], Goode and Kao [4], Gupta and Groll [5], Gupta [6], Fertig and Mann [7], Kantam and Rosaiah [8], Kantam et. al. [9], Baklizi and El Marsi [10], Rosaiah and Kantam [11], Tsai and $\mathrm{Wu}$ [12], Balakrishnan et. al. [13], Rao et. al. [14].

As it can be seen from previous studies, the acceptance sampling plans were developed based on mean life time under a truncated life test. Referring to the advantages over and above of a mean life test, Lio et. al. [15] considered acceptance sampling plans for percentiles using truncated life tests assuming the life times follows Birnbaum-Saunders distribution. The reason quoted for this approach is acceptance sampling plans based on the mean may not satisfy the requirement of engineering on the strength or breaking stress which can be better expressed by percentiles. When the required quality is given by a specified low percentile, an acceptance sampling plan based on the mean may accept the lot which has low percentile below the required standard of consumers. Further to add, a small decrease in the mean with a simultaneous small increase in the variance can result in a significant downward shift in a small percentile of interest. This means that a lot of products could be 
accepted due to a small decrease in the mean life time after inspection. But the material strengths of products may be devolving significantly and thus failed to meet the consumer's requirement. Therefore, engineers pay more attention to the percentiles of lifetimes than the mean lifetime in life testing applications. Moreover, the observed lifetime distributions are not symmetric. Referring to Marshall and Olkin [16], the mean lifetime may not be adequate to describe the central tendency of the distribution. This reduces the feasibility of acceptance sampling plans if they are developed based on the mean lifetime of products. Actually, percentiles provide more information regarding a lifetime distribution than what is provided by mean lifetime. When the lifetime distribution is symmetric, the $50^{\text {th }}$ percentile of the median is equivalent to the mean lifetime. Hence, developing acceptance sampling plans based on percentiles (ASPP) of a life time distribution can be treated as a generalization of developing acceptance sampling plans based on the mean lifetime of products. This type of acceptance sampling plans based on percentiles has proved their importance and these plans are developed for various distributions viz., Lio et. al. [15,17], Rao and Kantam [18], Rao [19], Rao et. al. [20,21] and the references therein.

The aim of the present study is to develop an ASPP for lot when the lifetime of a product follows Type-II generalized $\log$ logistic distribution (TGLLD). In this paper, the subsequent part of the study is arranged in the following way. Introduction of TGLLD is given in Section 2. In section 3, the design of proposed acceptance sampling plan based on percentiles for lifetimes under a truncated life test is described. In Section 4, description of the proposed methodology and illustrative real data example is given. Finally, conclusions drawn under this plan are given in Section 5.

\section{Type-II Generalized Log Logistic Distribution}

Log-logistic distribution (LLD) has proven its importance in quality control. Different types of acceptance sampling plans are developed for LLD. The cumulative distribution function (cdf) of the log-logistic distribution (LLD) is

$$
F(t ; \sigma, \theta)=\frac{(t / \sigma)^{\lambda}}{\left[1+(t / \sigma)^{\lambda}\right]} ; t>0 ; \sigma>0, \lambda>1 .
$$

Since the practical pertinence of generalized log-logistic distribution (GLLD) in diverse sectors, various authors have paid their attention in developing different types of acceptance sampling plans when the lifetime variate follows GLLD. An extension to this distribution named as Type-II generalized log-logistic distribution (TGLLD) introduced by Rosaiah et al. [1], its cumulative distribution function (cdf) is

$$
F(t ; \sigma, \theta)=1-\left[1+(t / \sigma)^{\lambda}\right]^{-\theta} ; t>0 ; \sigma, \theta>0, \lambda>1 \text {. }
$$

It may be noted that the distribution given in (2) is defined through the reliability oriented generalization of log-logistic distribution. In short, we call this as the Type-II generalized log-logistic distribution [Type-I generalized (exponentiated) log-logistic distribution is dealt with by Rosaiah et al. [22]]. The corresponding probability density function (pdf) is given by

$$
f(t ; \sigma, \theta, \lambda)=\frac{(t / \sigma)^{\lambda}}{\left[1+(t / \sigma)^{\lambda}\right]^{\theta+1}} ; t>0 ; \sigma, \theta>0, \lambda>1
$$

where $\sigma$ is the scale parameter, $\lambda$ and $\theta$ are shape parameters.

The 100qth percentile of the TGLLD is given by

$$
t_{q}=\sigma \eta_{q}, \text { where } \eta_{q}=\left[(1-q)^{-1 / \theta}-1\right]^{1 / \lambda} .
$$

Hence, for fixed values of $\theta=\theta_{0}$ and $\lambda=\lambda_{0}$, the quantile $t_{q}$ in equation (4) is the function of scale parameter $\sigma=\sigma_{0}$ i.e., $t_{q} \geq t_{q}^{0} \Leftrightarrow \sigma \geq \sigma_{0}$, where $\sigma_{0}=t_{q}^{0} / \eta_{q}$.

It may be noted that $\sigma_{0}$ depends on $\theta_{0}$ and $\lambda_{0}$ to draw the acceptance sampling plans for TGLLD, one may ascertain $t_{q} \geq t_{q}^{0}$ equivalently that $\sigma$ exceeds $\sigma_{0}$.

\section{Design of the Proposed Acceptance Sampling Plan}

Aim of this study is to obtain minimum sample size required to ensure a percentile life when the life test is terminated at a pre-specified time $t_{q}^{0}$ and when the observed number of failures does not exceed a given acceptance number. The operating procedure is to accept a lot only if the specified percentile of lifetime is established with pre-specified high probability $\alpha$, which is an indicator of consumer confidence. The life test experiment gets terminated at the time $(\mathrm{c}+1)^{\text {th }}$ failure is observed or at quantile time $t_{q}$ whichever is earlier. Acceptance sampling plans based on truncated life test for TGLLD was developed by Rao et. al. [23,24].

The probability of accepting a lot based on the number of failures from a sample of $\mathrm{n}$ items under a truncated life test at the time schedule $t_{q}$ is given by

$$
P_{a}(p)=\sum_{i=0}^{c}\left(\begin{array}{l}
n \\
i
\end{array}\right) p^{i}(1-p)^{n-i}
$$

where $\mathrm{n}$ is the sample size, $\mathrm{c}$ is the acceptance number and $p$ is the probability that an item is failed before the test termination time $t_{0}$. If the lifetime of the product follows TGLLD, then $p=F\left(t_{0}\right)$, in a convenient approach, to determine the experiment test termination time $t_{0}$ as $t_{0}=\delta_{q}^{0} t_{q}^{0}$ for a constant $\delta_{q}^{0}$ and the targeted $100 \mathrm{q}^{\text {th }}$ lifetime percentile, $t_{q}^{0}$, suppose $t_{q}$ is the true $100 \mathrm{q}^{\text {th }}$ lifetime percentile. Then, $\mathrm{p}$ can be rewritten as

$$
p=1-\left[1+\left(t_{0} / \sigma\right)^{\lambda}\right]^{-\theta}=1-\left[1+\left\{\left(\frac{\eta_{q} \delta_{q}^{0}}{t_{q} / t_{q}^{0}}\right)^{\lambda}\right\}\right]^{-\theta}
$$


In order to find the design parameters of the proposed acceptance sampling plan, we prefer the approach based on two points on the curve by considering the producer's and consumer's risk. In this approach, the quality level is measured through the ratio of its percentile lifetime to the true lifetime, $t_{q} / t_{q}^{0}$. To ensure and improve the quality of the products, producer may use the percentile ratios. The probability $(\alpha)$ of rejecting a good lot is called the producer's risk and the probability $(\beta)$ of accepting a bad lot is known as the consumer's risk. At the interest of both producer and consumer, the inference drawn from an acceptance sampling plan so developed may fulfill their specified risks. Producer requires the lot acceptance probability of at least $1-\alpha$ at the Acceptable Reliability Level (ARL), $p_{1}$, i.e., the producer wants that the lot to be accepted at different values of percentile ratios say for the values of $t_{q} / t_{q}^{0}=2,4,6,8,10$ given in (5), another hand, the consumer wants the Lot Tolerance Reliability Level (LTRL), $p_{2}$, i.e., consumer may reject the lot if $t_{q} / t_{q}^{0}=1$ according to the equation (5).

$$
\begin{gathered}
P_{a}\left(p_{1}\right)=\sum_{i=0}^{c}\left(\begin{array}{l}
n \\
i
\end{array}\right) p_{1}^{i}\left(1-p_{1}\right)^{n-i} \geq 1-\alpha \\
P_{a}\left(p_{2}\right)=\sum_{i=0}^{c}\left(\begin{array}{l}
n \\
i
\end{array}\right) p_{2}^{i}\left(1-p_{2}\right)^{n-i} \leq \beta
\end{gathered}
$$

Where $p_{1}$ and $p_{2}$ are given by

$$
\begin{aligned}
& p_{1}=1-\left[1+\left\{\left(\frac{\eta_{q} \delta_{q}^{0}}{t_{q} / t_{q}^{0}}\right)^{\lambda}\right\}\right]^{-\theta} \\
& \text { and } p_{2}=1-\left[1+\left(\eta_{q} \delta_{q}^{0}\right)^{\lambda}\right]^{-\theta} .
\end{aligned}
$$

Estimates of the parameters of the proposed plan for different values of shape parameters are obtained, for different combinations of $\lambda$ and $\theta$ viz. $(\theta=1.5, \lambda=2)$, $(\theta=2, \lambda=2)$ and $(\theta=2.5, \lambda=2.5)$ at producer's risk $\alpha=0.05$ and test termination time $t_{0}=\delta_{q}^{0} t_{q}^{0}$ with $\delta_{q}^{0}=1.0,1.5,2.0,2.5,3.0$ the parameters of the proposed acceptance sampling plans based on percentiles are estimated for $50^{\text {th }}$ percentiles at different confidence levels $\beta=0.25,0.10,0.05,0.01$. The plan parameters along with operating characteristic values are presented in Table 1 - Table 3 for $(\theta=1.5, \lambda=2),(\theta=2, \lambda=2)$ and $(\theta=2.5, \lambda=2.5)$. For the data under consideration given in section 4.2, the MLE's obtained for the plan parameters $\theta$ and $\lambda$ are $\hat{\theta}^{\prime}=2.0722$ and $\hat{\lambda}=1.4273$ which are presented in Table 4 . We noticed from Table 1 - Table 4

\begin{tabular}{|c|c|c|c|c|c|c|c|c|c|c|c|c|c|c|c|c|}
\hline \multirow{2}{*}{$\beta$} & \multirow{2}{*}{$t_{q} / t_{q}^{0}$} & \multicolumn{3}{|c|}{$\delta_{q}=1.0$} & \multicolumn{3}{|c|}{$\delta_{q}=1.5$} & \multicolumn{3}{|c|}{$\delta_{q}=2.0$} & \multicolumn{3}{|c|}{$\delta_{q}=2.5$} & \multicolumn{3}{|c|}{$\delta_{q}=3.0$} \\
\hline & & $c$ & $n$ & $P_{a}\left(p_{1}\right)$ & $c$ & $n$ & $P_{a}\left(p_{1}\right)$ & $c$ & $n$ & $P_{a}\left(p_{1}\right)$ & $c$ & $n$ & $P_{a}\left(p_{1}\right)$ & $c$ & $n$ & $P_{a}\left(p_{1}\right)$ \\
\hline \multirow{5}{*}{0.25} & 2 & 5 & 14 & 0.9685 & 6 & 11 & 0.9511 & 9 & 13 & 0.9539 & 12 & 15 & 0.9596 & 13 & 15 & 0.9527 \\
\hline & 4 & 1 & 5 & 0.9751 & 1 & 3 & 0.9650 & 2 & 4 & 0.9779 & 3 & 5 & 0.9802 & 2 & 3 & 0.9577 \\
\hline & 6 & 0 & 2 & 0.9526 & 1 & 3 & 0.9920 & 1 & 3 & 0.9769 & 1 & 2 & 0.9816 & 1 & 2 & 0.9655 \\
\hline & 8 & 0 & 2 & 0.9730 & 1 & 3 & 0.9973 & 1 & 3 & 0.9920 & 1 & 2 & 0.9936 & 1 & 2 & 0.9874 \\
\hline & 10 & 0 & 2 & 0.9826 & 0 & 2 & 0.9614 & 0 & 1 & 0.9658 & 1 & 2 & 0.9972 & 1 & 2 & 0.9945 \\
\hline \multirow{5}{*}{0.1} & 2 & 6 & 19 & 0.9522 & 9 & 17 & 0.9629 & 12 & 18 & 0.9519 & 17 & 22 & 0.9568 & - & - & - \\
\hline & 4 & 1 & 7 & 0.9512 & 2 & 6 & 0.9782 & 2 & 5 & 0.9524 & 3 & 5 & 0.9802 & 4 & 6 & 0.9782 \\
\hline & 6 & 1 & 7 & 0.9889 & 1 & 4 & 0.9845 & 1 & 3 & 0.9769 & 2 & 4 & 0.9911 & 2 & 4 & 0.9779 \\
\hline & 8 & 1 & 7 & 0.9963 & 1 & 4 & 0.9947 & 1 & 3 & 0.9920 & 1 & 3 & 0.9817 & 1 & 3 & 0.9651 \\
\hline & 10 & 0 & 4 & 0.9655 & 0 & 2 & 0.9614 & 1 & 3 & 0.9966 & 1 & 3 & 0.9920 & 1 & 3 & 0.9842 \\
\hline \multirow{5}{*}{0.05} & 2 & 7 & 23 & 0.9508 & 11 & 21 & 0.9698 & 14 & 21 & 0.9608 & - & - & - & - & - & - \\
\hline & 4 & 2 & 11 & 0.9825 & 2 & 7 & 0.9651 & 2 & 5 & 0.9524 & 3 & 6 & 0.9531 & 5 & 8 & 0.9753 \\
\hline & 6 & 1 & 8 & 0.9854 & 1 & 5 & 0.9751 & 1 & 4 & 0.9566 & 2 & 5 & 0.9799 & 2 & 4 & 0.9779 \\
\hline & 8 & 1 & 8 & 0.9951 & 1 & 5 & 0.9914 & 1 & 4 & 0.9845 & 1 & 3 & 0.9817 & 1 & 3 & 0.9650 \\
\hline & 10 & 0 & 5 & 0.9570 & 1 & 5 & 0.9963 & 1 & 4 & 0.9933 & 1 & 3 & 0.9920 & 1 & 3 & 0.9842 \\
\hline \multirow{5}{*}{0.01} & 2 & 10 & 35 & 0.9525 & 14 & 29 & 0.9543 & - & - & - & - & - & - & - & - & - \\
\hline & 4 & 2 & 14 & 0.9657 & 3 & 10 & 0.9809 & 3 & 8 & 0.9558 & 4 & 8 & 0.9643 & 6 & 10 & 0.9747 \\
\hline & 6 & 1 & 11 & 0.9726 & 1 & 6 & 0.9639 & 2 & 6 & 0.9880 & 2 & 5 & 0.9799 & 2 & 5 & 0.9524 \\
\hline & 8 & 1 & 11 & 0.9906 & 1 & 6 & 0.9874 & 1 & 5 & 0.9751 & 1 & 4 & 0.9654 & 2 & 5 & 0.9881 \\
\hline & 10 & 1 & 11 & 0.9960 & 1 & 6 & 0.9946 & 1 & 5 & 0.9891 & 1 & 4 & 0.9845 & 1 & 4 & 0.9701 \\
\hline
\end{tabular}
that percentile ratio increases the sample size $\mathrm{n}$ decreases for all the parameters combination.

Table 1. Minimum sample size required to ensure $50^{\text {th }}$ percentile and the respective OC values of TGLLD for $\theta=1.5$ and $\lambda=2$ 
Table 2. Minimum sample size required to ensure $50^{\text {th }}$ percentile and the respective $O C$ values of TGLLD for $\theta=2$ and $\lambda=2$

\begin{tabular}{|c|c|c|c|c|c|c|c|c|c|c|c|c|c|c|c|c|}
\hline \multirow{2}{*}{$\beta$} & \multirow{2}{*}{$t_{q} / t_{q}^{0}$} & \multicolumn{3}{|c|}{$\delta_{q}=1.0$} & \multicolumn{3}{|c|}{$\delta_{q}=1.5$} & \multicolumn{3}{|c|}{$\delta_{q}=2.0$} & \multicolumn{3}{|c|}{$\delta_{q}=2.5$} & \multicolumn{3}{|c|}{$\delta_{q}=3.0$} \\
\hline & & $c$ & $n$ & $P_{a}\left(p_{1}\right)$ & c & $n$ & $P_{a}\left(p_{1}\right)$ & $c$ & $n$ & $P_{a}\left(p_{1}\right)$ & $c$ & $n$ & $P_{a}\left(p_{1}\right)$ & $c$ & $n$ & $P_{a}\left(p_{1}\right)$ \\
\hline \multirow{5}{*}{0.25} & 2 & 4 & 12 & 0.9522 & 5 & 9 & 0.9519 & 8 & 11 & 0.9673 & 9 & 11 & 0.9528 & 14 & 16 & 0.9533 \\
\hline & 4 & 1 & 5 & 0.9776 & 1 & 3 & 0.9681 & 2 & 4 & 0.9802 & 2 & 3 & 0.9826 & 2 & 3 & 0.9599 \\
\hline & 6 & 1 & 5 & 0.9951 & 1 & 3 & 0.9928 & 1 & 3 & 0.9791 & 1 & 2 & 0.9832 & 1 & 2 & 0.9680 \\
\hline & 8 & 0 & 3 & 0.9620 & 1 & 3 & 0.9976 & 0 & 1 & 0.9502 & 1 & 2 & 0.9942 & 1 & 2 & 0.9885 \\
\hline & 10 & 0 & 3 & 0.9755 & 0 & 2 & 0.9636 & 0 & 1 & 0.9677 & 0 & 1 & 0.9502 & 1 & 2 & 0.9950 \\
\hline \multirow{5}{*}{0.1} & 2 & 6 & 19 & 0.9602 & 8 & 15 & 0.9634 & 11 & 16 & 0.9616 & 15 & 19 & 0.9577 & - & - & - \\
\hline & 4 & 1 & 7 & 0.9559 & 2 & 6 & 0.9809 & 2 & 4 & 0.9802 & 3 & 5 & 0.9821 & 3 & 5 & 0.9502 \\
\hline & 6 & 1 & 7 & 0.9900 & 1 & 4 & 0.9861 & 1 & 3 & 0.9791 & 1 & 3 & 0.9531 & 1 & 2 & 0.9680 \\
\hline & 8 & 1 & 7 & 0.9967 & 1 & 4 & 0.9953 & 1 & 3 & 0.9928 & 1 & 3 & 0.9834 & 1 & 2 & 0.9885 \\
\hline & 10 & 0 & 4 & 0.9675 & 0 & 2 & 0.9636 & 1 & 3 & 0.9969 & 0 & 1 & 0.9502 & 1 & 2 & 0.9950 \\
\hline \multirow{5}{*}{0.05} & 2 & 7 & 23 & 0.9596 & 10 & 19 & 0.9706 & 12 & 18 & 0.9517 & 18 & 23 & 0.9621 & - & - & - \\
\hline & 4 & 2 & 11 & 0.9849 & 2 & 6 & 0.9809 & 2 & 5 & 0.9570 & 3 & 6 & 0.9574 & 3 & 5 & 0.9502 \\
\hline & 6 & 1 & 8 & 0.9869 & 1 & 5 & 0.9776 & 1 & 4 & 0.9605 & 1 & 3 & 0.9539 & 2 & 4 & 0.9802 \\
\hline & 8 & 1 & 8 & 0.9956 & 1 & 5 & 0.9923 & 1 & 4 & 0.9860 & 1 & 3 & 0.9834 & 1 & 3 & 0.9681 \\
\hline & 10 & 0 & 5 & 0.9595 & 1 & 5 & 0.9967 & 17 & 4 & 0.9940 & 1 & 3 & 0.9928 & 1 & 3 & 0.9858 \\
\hline \multirow{5}{*}{0.01} & 2 & 10 & 35 & 0.9628 & 13 & 26 & 0.9689 & 3 & 26 & 0.9622 & - & - & - & - & - & - \\
\hline & 4 & 2 & 14 & 0.9702 & 2 & 8 & 0.9546 & 2 & 7 & 0.9774 & 3 & 6 & 0.9574 & 5 & 8 & 0.9775 \\
\hline & 6 & 1 & 11 & 0.9754 & 1 & 6 & 0.9674 & 1 & 6 & 0.9896 & 2 & 5 & 0.9822 & 2 & 5 & 0.9570 \\
\hline & 8 & 1 & 11 & 0.9916 & 1 & 6 & 0.9887 & 1 & 5 & 0.9776 & 1 & 4 & 0.9686 & 1 & 3 & 0.9681 \\
\hline & 10 & 1 & 11 & 0.9965 & 1 & 6 & 0.9952 & & 5 & 0.9902 & 1 & 4 & 0.9861 & 1 & 3 & 0.9858 \\
\hline
\end{tabular}

Table 3. Minimum sample size required to ensure $50^{\text {th }}$ percentile and the respective OC values of TGLLD for $\theta=2.5$ and $\lambda=2.5$

\begin{tabular}{|c|c|c|c|c|c|c|c|c|c|c|c|c|c|c|c|c|}
\hline \multirow{2}{*}{$\beta$} & \multirow{2}{*}{$t_{q} / t_{q}^{0}$} & \multicolumn{3}{|c|}{$\delta_{q}=1.0$} & \multicolumn{3}{|c|}{$\delta_{q}=1.5$} & \multicolumn{3}{|c|}{$\delta_{q}=2.0$} & \multicolumn{3}{|c|}{$\delta_{q}=2.5$} & \multicolumn{3}{|c|}{$\delta_{q}=3.0$} \\
\hline & & $c$ & $n$ & $P_{a}\left(p_{1}\right)$ & $c$ & $n$ & $P_{a}\left(p_{1}\right)$ & $c$ & $n$ & $P_{a}\left(p_{1}\right)$ & $c$ & $n$ & $P_{a}\left(p_{1}\right)$ & $c$ & $n$ & $P_{a}\left(p_{1}\right)$ \\
\hline \multirow{5}{*}{0.25} & 2 & 2 & 7 & 0.9504 & 4 & 7 & 0.9697 & 6 & 8 & 0.9648 & 7 & 8 & 0.9594 & 12 & 13 & 0.9503 \\
\hline & 4 & 0 & 2 & 0.9515 & 1 & 3 & 0.9877 & 1 & 2 & 0.9835 & 1 & 2 & 0.9561 & 2 & 3 & 0.9721 \\
\hline & 6 & 0 & 2 & 0.9821 & 0 & 1 & 0.9755 & 0 & 1 & 0.9505 & 1 & 2 & 0.9929 & 1 & 2 & 0.9835 \\
\hline & 8 & 0 & 2 & 0.9912 & 0 & 1 & 0.9879 & 0 & 1 & 0.9755 & 0 & 1 & 0.9577 & 1 & 2 & 0.9957 \\
\hline & 10 & 0 & 2 & 0.9950 & 0 & 1 & 0.9931 & 0 & 1 & 0.9859 & 0 & 1 & 0.9755 & 0 & 1 & 0.9617 \\
\hline \multirow{5}{*}{0.1} & 2 & 4 & 14 & 0.9744 & 5 & 9 & 0.9732 & 8 & 11 & 0.9673 & 11 & 13 & 0.9594 & - & - & - \\
\hline & 4 & 1 & 7 & 0.9884 & 1 & 4 & 0.9764 & 1 & 3 & 0.9548 & 1 & 2 & 0.9561 & 2 & 3 & 0.9721 \\
\hline & 6 & 0 & 4 & 0.9645 & 0 & 2 & 0.9515 & 0 & 1 & 0.9505 & 1 & 2 & 0.9929 & 1 & 2 & 0.9835 \\
\hline & 8 & 0 & 4 & 0.9825 & 0 & 2 & 0.9760 & 0 & 1 & 0.9755 & 0 & 1 & 0.9577 & 1 & 2 & 0.9957 \\
\hline & 10 & 0 & 4 & 0.9900 & 0 & 2 & 0.9862 & 0 & 1 & 0.9859 & 0 & 1 & 0.9755 & 0 & 1 & 0.9617 \\
\hline \multirow{5}{*}{0.05} & 2 & 4 & 16 & 0.9551 & 5 & 10 & 0.9501 & 8 & 11 & 0.9673 & 14 & 17 & 0.9532 & - & - & - \\
\hline & 4 & 1 & 8 & 0.9847 & 1 & 4 & 0.9764 & 1 & 3 & 0.9548 & 2 & 4 & 0.9689 & 2 & 3 & 0.9721 \\
\hline & 6 & 0 & 5 & 0.9558 & 0 & 2 & 0.9515 & 1 & 3 & 0.9929 & 1 & 3 & 0.9799 & 1 & 2 & 0.9835 \\
\hline & 8 & 0 & 5 & 0.9782 & 0 & 2 & 0.9760 & 0 & 2 & 0.9515 & 0 & 1 & 0.9577 & 1 & 2 & 0.9957 \\
\hline & 10 & 0 & 5 & 0.9875 & 0 & 2 & 0.9862 & 0 & 2 & 0.9719 & 0 & 1 & 0.9755 & 0 & 1 & 0.9617 \\
\hline \multirow{5}{*}{0.01} & 2 & 6 & 25 & 0.9663 & 8 & 16 & 0.9723 & 11 & 16 & 0.9616 & 17 & 21 & 0.9507 & - & - & - \\
\hline & 4 & 1 & 11 & 0.9714 & 1 & 5 & 0.9623 & 2 & 5 & 0.9827 & 2 & 4 & 0.9689 & 3 & 5 & 0.9679 \\
\hline & 6 & 1 & 11 & 0.9958 & 1 & 5 & 0.9943 & 1 & 4 & 0.9863 & 1 & 3 & 0.9799 & 1 & 3 & 0.9548 \\
\hline & 8 & 0 & 7 & 0.9696 & 0 & 3 & 0.9643 & 0 & 2 & 0.9515 & 1 & 3 & 0.9948 & 1 & 3 & 0.9877 \\
\hline & 10 & 0 & 7 & 0.9825 & 0 & 3 & 0.9794 & 0 & 2 & 0.9717 & 0 & 2 & 0.9515 & 1 & 3 & 0.9957 \\
\hline
\end{tabular}


Table 4. Minimum sample size required to ensure $50^{\text {th }}$ percentile and the respective OC values of TGLLD for $\hat{\theta}=2.0722$ and $\hat{\lambda}=1.4273$

\begin{tabular}{|c|c|c|c|c|c|c|c|c|c|c|c|c|c|c|c|c|}
\hline \multirow{2}{*}{$\beta$} & \multirow{2}{*}{$t_{q} / t_{q}^{0}$} & \multicolumn{3}{|c|}{$\delta_{q}=1.0$} & \multicolumn{3}{|c|}{$\delta_{q}=1.5$} & \multicolumn{3}{|c|}{$\delta_{q}=2.0$} & \multicolumn{3}{|c|}{$\delta_{q}=2.5$} & \multicolumn{3}{|c|}{$\delta_{q}=3.0$} \\
\hline & & $c$ & $n$ & $P_{a}\left(p_{1}\right)$ & $c$ & $n$ & $P_{a}\left(p_{1}\right)$ & $c$ & $n$ & $P_{a}\left(p_{1}\right)$ & $c$ & $n$ & $P_{a}\left(p_{1}\right)$ & $c$ & $n$ & $P_{a}\left(p_{1}\right)$ \\
\hline \multirow{5}{*}{0.25} & 2 & 9 & 23 & 0.9609 & 10 & 18 & 0.9571 & 12 & 18 & 0.9519 & 15 & 20 & 0.9538 & 17 & 21 & 0.9503 \\
\hline & 4 & 2 & 7 & 0.9708 & 2 & 5 & 0.9588 & 2 & 4 & 0.9501 & 3 & 5 & 0.9617 & 4 & 6 & 0.9659 \\
\hline & 6 & 1 & 5 & 0.9672 & 2 & 5 & 0.9902 & 2 & 4 & 0.9876 & 2 & 4 & 0.9727 & 2 & 4 & 0.9501 \\
\hline & 8 & 1 & 5 & 0.9848 & 1 & 4 & 0.9722 & 1 & 3 & 0.9693 & 2 & 4 & 0.9802 & 1 & 2 & 0.9690 \\
\hline & 10 & 1 & 5 & 0.9915 & 1 & 4 & 0.9845 & 1 & 3 & 0.9827 & 1 & 3 & 0.9693 & 1 & 2 & 0.9823 \\
\hline \multirow{5}{*}{0.1} & 2 & 12 & 33 & 0.9539 & 15 & 28 & 0.9658 & 18 & 28 & 0.9564 & - & - & - & - & - & - \\
\hline & 4 & 3 & 12 & 0.9700 & 3 & 8 & 0.9631 & 4 & 8 & 0.9735 & 4 & 7 & 0.9628 & 5 & 8 & 0.9594 \\
\hline & 6 & 2 & 9 & 0.9856 & 2 & 6 & 0.9819 & 2 & 5 & 0.9724 & 3 & 6 & 0.9830 & 2 & 4 & 0.9501 \\
\hline & 8 & 1 & 7 & 0.9692 & 1 & 5 & 0.9559 & 2 & 5 & 0.9902 & 2 & 5 & 0.9780 & 2 & 4 & 0.9811 \\
\hline & 10 & 1 & 7 & 0.9828 & 1 & 5 & 0.9750 & 1 & 4 & 0.9672 & 1 & 3 & 0.9693 & 1 & 3 & 0.9517 \\
\hline \multirow{5}{*}{0.05} & 2 & 15 & 42 & 0.9606 & 17 & 33 & 0.9563 & - & - & - & - & - & - & - & - & - \\
\hline & 4 & 3 & 13 & 0.9602 & 4 & 11 & 0.9694 & 4 & 9 & 0.9524 & 5 & 9 & 0.9661 & 5 & 8 & 0.9594 \\
\hline & 6 & 2 & 11 & 0.9742 & 2 & 7 & 0.9708 & 2 & 6 & 0.9510 & 3 & 7 & 0.9665 & 3 & 6 & 0.9633 \\
\hline & 8 & 1 & 8 & 0.9600 & 1 & 5 & 0.9559 & 2 & 6 & 0.9819 & 2 & 5 & 0.9780 & 2 & 5 & 0.9588 \\
\hline & 10 & 1 & 8 & 0.9775 & 1 & 5 & 0.9750 & 1 & 4 & 0.9672 & 2 & 5 & 0.9902 & 1 & 3 & 0.9517 \\
\hline \multirow{5}{*}{0.01} & 2 & - & - & - & - & - & - & - & - & - & - & - & - & - & - & - \\
\hline & 4 & 4 & 19 & 0.9579 & 5 & 15 & 0.9649 & 6 & 14 & 0.9630 & 7 & 13 & 0.9738 & 7 & 12 & 0.9550 \\
\hline & 6 & 2 & 14 & 0.9504 & 3 & 11 & 0.9782 & 3 & 9 & 0.9646 & 4 & 9 & 0.9803 & 4 & 8 & 0.9735 \\
\hline & 8 & 2 & 14 & 0.9821 & 2 & 9 & 0.9779 & 2 & 7 & 0.9703 & 2 & 6 & 0.9605 & 3 & 7 & 0.9786 \\
\hline & 10 & 1 & 11 & 0.9584 & 2 & 9 & 0.9903 & 2 & 7 & 0.9870 & 2 & 6 & 0.9819 & 2 & 6 & 0.9656 \\
\hline
\end{tabular}

\section{Description of the Proposed Methodology with Real Data Set}

\subsection{Description of the Proposed Plan}

Suppose a manufacturer wants to improve the quality of the manufactured product and the lifetime of the product follows the TGLLD with shape parameters $\theta=2$ and $\lambda=2$. Assume that the sampling plan is designed to ensure the $50^{\text {th }}$ percentile lifetime of the product is at least 1000 hours when $\beta=0.10$ and $\alpha=0.05$ at the percentile ratio $t_{q} / t_{q}^{0}$. We may find the optimal plan for this experiment from Table 2 with the given quality characteristics $\alpha=0.05, \beta=0.10, \quad \theta=2, \lambda=2$, $t_{q} / t_{q}^{0}=2$ and $\delta_{q}=1.0$ is found as $n=19$ and $c=6$ with acceptance probability 0.9602 .

The present study deals the sampling plan based on two-points on the OC curve approach to ensure percentile lifetime of the product when the lifetimes follows TGLLD instead the majority of the sampling plans developed for various lifetime distributions are based on one point OC curve approach for assuring average or percentile lifetime except Balamurali et.al. [25].

\subsection{Real Data Example}

In this subsection, we use a real data set to demonstrate the sampling plan and suitability of our distribution, TGLLD. We examine a data set corresponding to remission times (in months) of a random sample of 128 bladder cancer patients. This data set was previously studied by Lee and Wang [26], Lemonte and Cordeiro [27] and Zea et al. [28]. The data are given as follows:

$0.08,0.20,0.40,0.50,0.51,0.81,0.90,1.05,1.19,1.26$, $1.35,1.40,1.46,1.76,2.02,2.02,2.07,2.09,2.23,2.26$, $2.46,2.54,2.62,2.64,2.69,2.69,2.75,2.83,2.87,3.02$, $3.25,3.31,3.36,3.36,3.48,3.52,3.57,3.64,3.70,3.82$, $3.88,4.18,4.23,4.26,4.33,4.34,4.40,4.50,4.51,4.87$, 4.98, 5.06, 5.09, 5.17, 5.32, 5.32, 5.34, 5.41, 5.41, 5.49, $5.62,5.71,5.85,6.25,6.54,6.76,6.93,6.94,6.97,7.09$, $7.26,7.28,7.32,7.39,7.59,7.62,7.63,7.66,7.87,7.93$, $8.26,8.37,8.53,8.65,8.66,9.02,9.22,9.47,9.74,10.06$, $10.34,10.66,10.75,11.25,11.64,11.79,11.98,12.02$, $12.03,12.07,12.63,13.11,13.29,13.80,14.24,14.76$, $14.77,14.83,15.96,16.62,17.12,17.14,17.36,18.10$, $19.13,20.28,21.73,22.69,23.63,25.74,25.82,26.31$, $32.15,34.26,36.66,43.01,46.12,79.05$.

We applied Kolmogorov-Smirnov (KS) test statistic to test the goodness of fit of our model. The maximum likelihood estimates of the three parameter TGLLD for the remission times are $\hat{\sigma}=12.0449, \hat{\lambda}=1.4273$ and $\hat{\theta}=2.0722$, using the K-S test, it is found that the maximum distance D is 0.0351 with p-value is 0.9975 . We find that the TGLLD with three parameters provides a good fit for the remission times. Figure 1 depicts the plots of the histogram superimposed by fitted density and the Q-Q plot respectively. Hence, We conform that the TGLLD with three parameters provides a good fit for the remission times data set. 
Histogram and theoretical densities

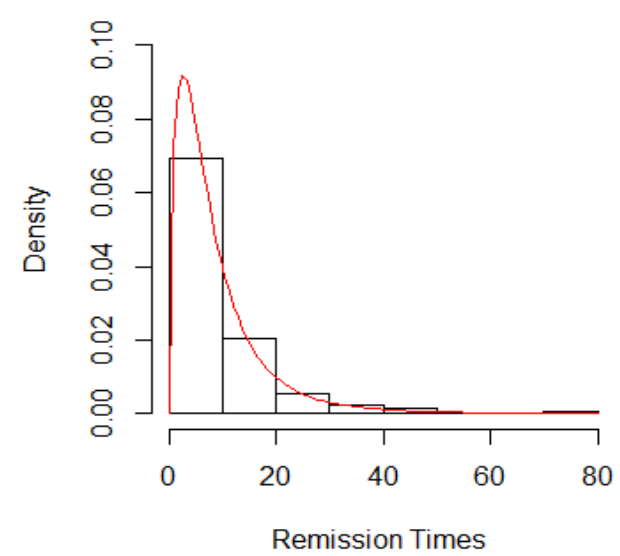

Q-Q plot

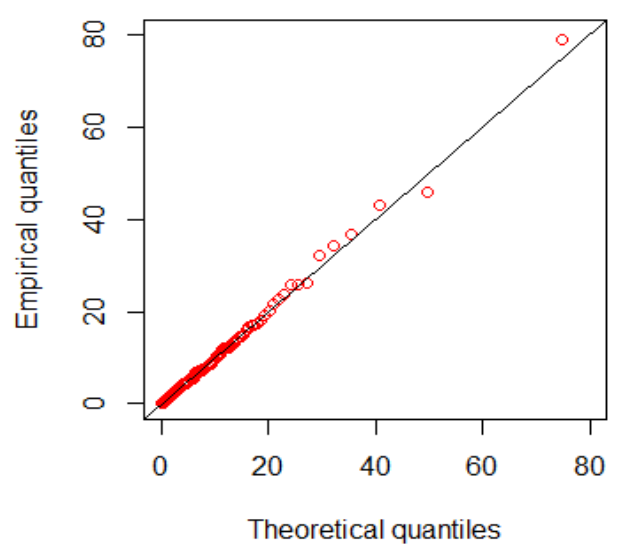

Figure 1. The density plot and Q-Q plot of the fitted TGLLD for the remission times data

Suppose, it is desired to develop a single acceptance sampling plan to satisfy the requirement that the $50^{\text {th }}$ percentile lifetime is greater than remission times 0.40 through the experiment to be completed by remission times 0.40 . Let us fix the consumer's risk at $25 \%$ when the true $50^{\text {th }}$ percentile is remission times 0.40 and the producer's risk at $5 \%$ when the true $50^{\text {th }}$ percentile is remission times 0.80 . Since $\hat{\lambda}=1.4273$ and $\hat{\theta}=2.0722$ the consumer's risk is $25 \%, \delta_{q}=1.5$ and $t_{q} / t_{q}^{0}=2$, the minimum sample size and acceptance number given by $n=18$ and $c=10$ from Table 4 . Thus the design can be implemented as follows. Selecting a sample of 18 remission times, we will accept the lot when no more than two failures occurs before remission times 0.80 . According to this plan, the remission times could have been accepted because there are only five failures before the termination time, remission times 0.80 .

\section{Conclusion}

In this article, a new acceptance sampling plan based on percentiles has been developed for the type-II generalized $\log$ logistic distribution assuming product having fixed tenure life and hence the life test is truncated with the end of product lifetime. This plan developed with a view to ensure the product life better than the specified one, hence considered the percentile ratio of lifetimes, the design parameters $\mathrm{c}$ and $\mathrm{n}$ of the proposed sampling plan are determined by the two-points on the OC curve approach method. Extensive tables have been provided for the industrial use according to various parameters and percentile values. It has been noticed that the proposed plan is better than the ordinary sampling plan with respect to sample size required for inspection. The methodology illustrated with real data set.

\section{References}

[1] Rosaiah, K., Kantam, R.R.L., Prasad, S.V.S.V.S.V., and Pratapa Reddy, J., "Reliability estimation in Type-II generalized loglogistic distribution," International Journal of Agricultural and Statistical Science, 4(2), 283-292, 2008.
[2] Epstein, B., "Truncated life tests in the exponential case," Annals of Mathematical Statistics, 25, 555-564, 1954.

[3] Sobel, M. and Tischendrof, J.A., "Acceptance Sampling with new life test objectives," Proceedings of fifth National Symposium on Reliability and Quality Control, Philadelphia Pennsylvania. 108-118, 1959.

[4] Goode, H.P. and Kao, J.H.K., "Sampling plans based on the Weibull distribution, Proceeding of Seventh National Symposium on Reliability and Quality Control," Philadelphia, Pennsylvania, 24-40, 1961.

[5] Gupta, S.S. and Groll, P.A., "Gamma distribution in acceptance sampling based on life tests," J. Amer. Statist. Assoc., 56, 942-970, 1961.

[6] Gupta, S.S., "Life test sampling plans for normal and lognormal distribution," Technometrics, 4, 151-175, 1962.

[7] Fertig, F.W. and Mann, N.R., "Life-test sampling plans for twoparameter Weibull populations," Technometrics, 22, 165-177, 1980.

[8] Kantam, R.R.L. and Rosaiah, K., "Half logistic distribution in acceptance sampling based on life tests," IAPQR Transactions, 23 (2), 117-125, 1998.

[9] Kantam, R. R. L., Rosaiah, K., and Rao, G. S. "Acceptance sampling based on life tests: log-logistic models," J. Appl. Statist., 28, 121-128, 2001.

[10] Baklizi, A and EI Masri, A.E.K., "Acceptance sampling based on truncated life tests in the Birnbaum-Saunders model," Risk Anal., 24, 1453-1457, 2004.

[11] Rosaiah, K and Kantam, R.R.L., "Acceptance sampling based on the inverse Rayleigh distribution," Economic Quality Control, 20, 277-286, 2005.

[12] Tsai, T.-R. and Wu, S.-J. "Acceptance sampling based on truncated life tests for generalized Rayleigh distribution," Journal of Applied Statistics, 33, 595-600, 2006.

[13] Balakrishnan, N. Leiva, V. and Lope, J., "Acceptance sampling plans from truncated life tests based on the generalized BirnbaumSaunders distribution," Comm. Statist. Simulation Comput., 36, 643-656, 2007.

[14] Rao, G.S., Ghitany, M.E. and Kantam, R.R.L., "Acceptance Sampling plans for Marshall-Olkin extented Lomax distribution," Int. J. Appl. Math., 21, 315-325, 2008.

[15] Lio, Y. L., Tsai, T.-R. and Wu, S.-J., "Acceptance sampling plans from truncated life tests based on the Burr type XII percentiles," $J$. Chinese Inst. Indust. Eng., 27, 270-280, 2010.

[16] Marshall, A.W and Olkin, I., Life Distributions -Structure of Nonparametric, Semiparametri and Parametric Families, Springer, New York, 2007.

[17] Lio, Y. L., Tsai, T.-R. and Wu, S.-J., “Acceptance sampling plan based on the truncated life test in the Birnbaum Saunders distribution for percentiles," Comm. Statist. Simulation Comput., 39, 119-136, 2009.

[18] Rao, G.S. and Kantam. R.R.L., "Acceptance Sampling Plans from Truncated Life Tests based on the Log-Logistic Distributions for Percentiles," Economic Quality Control, 25, 153-167, 2010. 
[19] Rao, G. S., "Acceptance sampling plans for percentiles based on the Marshall-Olkin extended Lomax distribution," Int. J. Stat. Econ., 11, 83-96, 2013.

[20] Rao, G.S., Rosaiah. K., Sridharbabu, M. and Sivakumar, D.C.U., "New Acceptance Sampling Plans Based on Percentiles for Exponentiated Frechet Distribution," Economic Quality Control, $31(1), 37-44,2016$

[21] Rao, G.S., Rosaiah. K., Kalyani K. and Sivakumar, D.C.U., "New Acceptance Sampling Plans Based on Percentiles for Odds Exponential Log Logistic Distribution," The Open Statistics \& Probability Journal, 7, 45-52, 2016.

[22] Rosaiah, K., Kantam, R.R.L., \& Santosh Kumar, Ch., "Reliability of test plans for exponentiated log-logistic distribution," Economic Quality Control, 21(2), 165-175, 2006.

[23] Rao, G. S., Kantam, R.R.L., Rosaiah, K. and Prasad, S.V.S.V.S.V., "Reliability test plans for type-II generalized log-logistic distribution," JRSS, , 5, 55-64, 2012.
[24] Rao, G. S., Kantam, R.R.L. Rosaiah. K. and Prasad, S.V.S.V.S.V., "An economic reliability test plan for generalized log-logistic distribution," International Journal of Engineering and Applied Sciences, 3, 61-68, 2012.

[25] Balamurali, S., Aslam, M. and Fallah, Nezhad, M.S., "An acceptance sampling plan under Fréchet distribution assuring median life," Research Journal of Applied Sciences, Engineering and Technology, 6(24), 4519-4523, 2013.

[26] Lee, E.T. and Wang, J.W., Statistical Methods for Survival Data Analysis, 3. Wiley-Interscience, Oklahoma, 2003.

[27] Lemonte, A.L. and Cordeiro, G. M., "An extended Lomax distribution, Statistics," A Journal of Theoretical and Applied Statistics, 1-17, 2011.

[28] Zea, L.M., Silva, R.B, Bourguignon, M., Santos, A.M. and Cordeiro, G.M., "The Beta Exponentiated Pareto Distribution with Application to Bladder Cancer Susceptibility," International Journal of Statistics and Probability, 2, 1927-7032, 2012. 